\title{
Clinical relevance of Mycobacterium malmoense isolation in the Netherlands
}

\author{
W. Hoefsloot*, J. van Ingen*,\#, W.C.M. de Lange*, P.N.R. Dekhuijzen*, \\ M.J. Boeree* and D. van Soolingen ${ }^{\#}$
}

ABSTRACT: Uncertainty exists about the clinical relevance of Mycobacterium malmoense isolation, especially in pulmonary samples. We therefore determined clinical relevance, treatment and outcome of $M$. malmoense isolation in the Netherlands.

A retrospective medical file study was conducted for all patients in the Netherlands from whom Mycobacterium malmoense had been isolated between January 2002 and January 2006. Diagnostic criteria for nontuberculous mycobacterial (NTM) disease published by the American Thoracic Society (ATS) were used to determine clinical relevance. Treatment was compared with guidelines published by the British Thoracic Society.

In total, 51 patients were found from whom M. malmoense was isolated. Of these, 40 (78\%) patients had pulmonary isolates and $32(80 \%)$ of them met the ATS diagnostic criteria. Cavitary disease was most common $(n=28 ; 88 \%)$. Patients with pulmonary disease were mostly males, with an average age of $\mathbf{5 6}$ yrs and pre-existing chronic obstructive pulmonary disease. Cervical lymphadenitis was the most common extrapulmonary disease type. Adherence to treatment guidelines was poor. A good clinical response to treatment was observed in $70 \%$ and $73 \%$ of patients treated for pulmonary and extrapulmonary disease, respectively.

In conclusion, M. malmoense is a clinically highly relevant NTM in the Netherlands causing serious pulmonary morbidity. Adherence to treatment guidelines is not satisfactory.

KEYWORDS: Atypical mycobacteria, atypical mycobacterium infections, Mycobacterium
malmoense

\section{AFFILIATIONS}

${ }^{*}$ Dept of Pulmonary Diseases, Radboud University Nijmegen Medical Centre, Nijmegen, and \#National Mycobacteria Reference Laboratory, National Institute for Public Health and the Environment, Bilthoven, The Netherlands.

\section{CORRESPONDENCE}

W. Hoefsloot

Radboud University Nijmegen

Medical Center

Dept of Pulmonary Diseases (454)

P.0. Box 9101

6500 HB Nijmegen

The Netherlands

E-mail: w.hoefsloot@long.umcn.nl

Received:

March 092009

Accepted after revision:

April 092009

First published online:

April 222009 irst described as a respiratory tract pathogen in 1977 by SCHRÖDER and JUHLIN [1], Mycobacterium malmoense is among the most frequently isolated and clinically relevant nontuberculous mycobacteria (NTM) in northern Europe [2-4]. The environment is the suspected source of transmission of NTM to humans through aerosols and ingestion. Person-to-person transmission or transmission from animal sources has not been proven $[3,5,6]$. The presence of NTM in the environment implies that a NTM cultured from a nonsterile body site, such as the respiratory tract, may result from contamination or occasional presence of the NTM in a sample. Hence, it is important to distinguish contamination from true NTM disease. The American Thoracic Society (ATS) has published guidelines to assist in this distinction [3]. The clinical relevance of a NTM species can be quantified by assessing the percentage of patients with positive cultures of the respective NTM who meet the ATS diagnostic criteria.

In the current study, we quantified the clinical relevance of $M$. malmoense isolation in the
Netherlands between 2002 and 2006 by applying the ATS diagnostic criteria, and evaluated treatment and outcome.

\section{MATERIALS AND METHODS \\ Study subjects}

Patients were identified by reviewing the database of the Dutch National Institute for Public Health and the Environment (RIVM; Bilthoven, the Netherlands) for $M$. malmoense positive cultures. The RIVM is the national mycobacteria reference laboratory that provides identification and drug susceptibility testing for all hospitals in the Netherlands. We reviewed medical records of the identified patients from whom $M$. malmoense was cultured between January 2002 and January 2006.

\section{Study design and setting}

The present study was a retrospective observational study concerning all patients with M. malmoense positive cultures identified at the RIVM. The Central Committee on Research involving Human Subjects, Arnhem-Nijmegen regional office (Radboud University Nijmegen
European Respiratory Journal Print ISSN 0903-1936 Online ISSN 1399-3003 
Medical Center, Nijmegen, the Netherlands) approved the study. We recorded demographic data, clinical data, drug susceptibility, treatment and outcome. The 2007 ATS diagnostic criteria were used to determine clinically relevant infections (table 1) [3].

Treatment was compared with guidelines published by the British Thoracic Society (BTS): a NTM-based treatment regimen was defined as consisting of rifampicin or rifabutin and ethambutol [7]. An adequate response to treatment was defined as symptomatic improvement and reversion to at least three subsequent negative cultures.

The RIVM subjected isolates of most patients to laboratory diagnosis. All patients' isolates were subcultured in both liquid and solid media and identified using the Inno-Lipa Mycobacteria v2 (Innogenetics, Ghent, Belgium) reverse line blot assay, which has specific probes for M. malmoense. Prior to 2004, 16S rDNA gene sequencing (151 bp hypervariable region A) was performed, after ruling out membership of the Mycobacterium tuberculosis or Mycobacterium avium complex using the AccuProbe assays (GenProbe, San Diego, CA, USA). Remaining isolates were identified at local hospitals, by $16 \mathrm{~S}$ sequencing.

Drug susceptibility was tested using the agar dilution method [8]. Drugs included in the test panel were isoniazid, rifampicin, ethambutol, streptomycin, cycloserine, prothionamide, amikacin, ciprofloxacin, clofazimine, clarithromycin and rifabutin.

\section{Data analysis}

The Pearson Chi-squared and Fisher exact tests were used for statistical correlations.

\section{RESULTS}

M. malmoense was isolated from 51 patients in the study period. In total, 40 (78\%) patients had pulmonary isolates; in $11(22 \%)$ cases these were of extrapulmonary origin. No patients in the study group were HIV infected.

During the study period, no increase in notification of M. malmoense isolation was observed each year.

\section{Pulmonary isolates}

Of all 40 patients with pulmonary M. malmoense isolates, 32 $(80 \%)$ met the ATS diagnostic criteria and were likely to suffer
M. malmoense lung disease. The baseline patient characteristics are detailed in table 2 .

The predominant patient profile is a male with pre-existing pulmonary disease, mainly chronic obstructive pulmonary disease. The seven patients without a previous diagnosis of pre-existing pulmonary disease were mostly smokers, with radiographic features suggestive of pulmonary disease. Most patients reported productive cough $(n=37 ; 93 \%)$, weight loss $(\mathrm{n}=24 ; 60 \%)$ and fatigue $(23 ; 58 \%)$. Night sweats $(\mathrm{n}=10 ; 25 \%)$ haemoptysis $(n=7 ; 8 \%)$ or fever $(n=11 ; 28 \%)$ were infrequently reported. Only patients who reported weight loss were more likely to meet the ATS diagnostic criteria $(p=0.048$; odds ratio $7.333 ; 95 \%$ confidence interval 1.072-50.145). In the group of patients that did not meet the ATS diagnostic criteria, four failed to meet the bacteriological criteria (three because only one sputum sample was collected) and four failed to meet the bacteriological and radiological criteria. Overall, 75\% $(n=24)$ of the 32 patients that met the ATS criteria for pulmonary NTM disease presented with cavitary lesions visible on chest radiographs. Additional computed tomography scanning revealed four extra cases of cavitary disease (total $n=28$; $88 \%$ ), not identified as such using plain chest radiographs. Two patients presented with multiple nodular opacities on chest radiograph; two had a single pulmonary mass.

The 30 patients who met the ATS diagnostic criteria for pulmonary NTM disease started treatment. Figure 1 summarises treatment and outcome in the study group. The mean duration of antimycobacterial treatment was 12 months (range 1-26 months). Macrolides were added in 22 patients (18 clarithromycin and four azithromycine; 92\%), fluoroquinolones in six patients (four ciprofloxacin and two moxifloxacin; $25 \%$ ). Nine patients received therapy for presumed tuberculosis, prior to the diagnosis of NTM disease, for a mean duration of 48 days (range 2-123 days), and completed a NTM-based regimen afterwards. Six patients with M. malmoense pulmonary disease only received a complete first-line tuberculosis treatment.

Of the 30 patients treated, $21(70 \%)$ patients showed an adequate response, five $(17 \%)$ suffered a failure or relapse (mean time to relapse 13 months, range 5-24 months) and four $(13 \%)$ died (fig. 1). Although the percentage of patients with an adequate response was lower in those receiving macrolidecontaining regimens (43 versus $63 \%$ ), this difference was not

\footnotetext{
TABLE 1 Summary of the 2007 American Thoracic Society diagnostic criteria of nontuberculous mycobacterial (NTM) lung disease

Clinical criteria

1. Pulmonary symptoms, nodular or cavitary opacities on chest radiograph, or an HRCT scan that shows multifocal bronchiectasis with multiple small nodules; and

2. Appropriate exclusion of other diagnoses.

Microbiological criteria

1. Positive culture results from at least two separate expectorated sputum samples (if the results from the initial sputum samples are nondiagnostic, consider repeat sputum AFB smears and cultures); or

2. Positive culture results from at least one bronchial wash or lavage; or

3. Transbronchial or other lung biopsy with mycobacterial histopathological features (granulomatous inflammation or AFB) and positive culture for NTM or biopsy

showing mycobacterial histopathological features (granulomatous inflammation or AFB) and one or more sputum or bronchial washings that are culture positive for NTM

HRCT: high-resolution computed tomography; AFB: acid-fast bacilli.
} 


\begin{tabular}{|c|c|c|c|c|}
\hline & ATS criteria met & ATS criteria not met & Total & $\mathrm{p}$-value \\
\hline Males & $21(66)$ & 7 (88) & 28 & 0.67 \\
\hline Age yrs & $56(28-81)$ & 57 (33-83) & $56(28-83)$ & 0.84 \\
\hline Prior TB & $2(6)$ & $2(25)$ & 4 & 0.18 \\
\hline AFB smear positive & $27(84)$ & $2(25)$ & 29 & 0.03 \\
\hline Cavitary lesion & $28(88)$ & $1(13)$ & 29 & $<0.001$ \\
\hline Nodular lesion(s) & $4(13)$ & $1(13)$ & 5 & 0.74 \\
\hline
\end{tabular}

Data are presented as $n$ (\%) or mean (range), unless otherwise stated. ATS: American Thoracic Society; COPD: chronic obstructive pulmonary disease; TB: tuberculosis; AFB: acid-fast bacilli.

statistically significant $(p=0.344)$. The mean duration of treatment among patients who later relapsed was shorter than for patients with an adequate response, although not significantly (320 versus 358 days; $\mathrm{p}=0.709$ ). The frequency of adequate response was not significantly different between patients treated with a tuberculosis-based regimen and those treated with a NTM regimen $(p=0.260)$. Two patients who met the ATS criteria refused treatment: one patient died, the other showed progressive disease. Follow-up of patients not meeting ATS diagnostic criteria was uneventful; no more positive cultures were recorded. Symptoms regressed in the absence of antimycobacterial treatment.

Six $(20 \%)$ patients received the 24 months of rifampicin and ethambutol regimen based on the BTS trials. This did not affect the percentage of patients with an adequate response $(83 \%$ for the BTS regimen versus $71 \%$ for other regimens; $\mathrm{p}=0.426$ ).

Contact-tracing studies were initiated for two patients with pulmonary $M$. malmoense isolates; both were presumed to have pulmonary tuberculosis. Some contacts received 6 months of isoniazid, based on a tuberculin skin test conversion.

In the current study, one case of disseminated M. malmoense disease was noted in a patient who received immunosuppressive treatment after kidney transplantation. He presented with pulmonary M. malmoense disease, which extended to histologically and bacteriologically proven lymphadenitis and mediastinooesophageal fistula, with blood cultures yielding M. malmoense. Interestingly, this patient had strong epidemiological links to a patient diagnosed with smear-positive pulmonary $M$. malmoense disease 1 yr before.

\section{Extrapulmonary isolates}

Eleven patients had extrapulmonary $M$. malmoense isolates; we noted 10 cases of cervicofacial lymphadenitis, including two in elderly patients. One case of tenosynovitis of the second and third digit of the right hand was observed in a plant handler with a history of multiple wounds to the right wrist. He had an adequate response after surgical debridement followed by a macrolide-based regimen of 13 months' duration. The eight paediatric cases of lymphadenitis were three males and five females without predisposing conditions, with a mean age of 36 months (range 22-46 months). All presented with painless cervical or submandibular swelling, without fever or other symptoms. Surgical excision was the most frequent treatment and resulted in an adequate response in all patients. One of the elderly patients had a relapse after surgery; the other had an adequate response. Overall, eight $(73 \%)$ patients with extrapulmonary isolates had an adequate response after the initial therapy.

In vitro drug susceptibility testing was performed on the primary isolates from 46 patients. Isolates were resistant to isoniazid (all), streptomycin (70\%), amikacin (70\%) and ciprofloxacin (61\%); intermediately susceptible (39\%) or resistant $(46 \%)$ to ethambutol; and susceptible to rifampicin $(72 \%)$, rifabutin $(96 \%)$, clarithromycin (all), cycloserine $(98 \%)$, prothionamide (96\%) and clofazimine (all).

Relapse or treatment failure among patients with pulmonary M. malmoense disease was not associated with in vitro rifampicin or ethambutol resistance $(p=0.327$ and $p=0.405$, respectively).

\section{DISCUSSION}

M. malmoense is one of the most clinically relevant NTM in the Netherlands. Overall, $80 \%$ of patients with pulmonary isolates met the ATS diagnostic criteria, compared with $21(47 \%)$ relevant infections among 45 patients with pulmonary M. xenopi isolates, and 11 (73\%) among 15 patients with pulmonary $M$. szulgai isolates $[9,10]$. We observed M. malmoense disease exclusively in HIV-negative patients, which is in contrast to $M$. avium, M. kansasii, $M$. xenopi and $M$. szulgai $[3,9,10]$. Patients were mainly males with pre-existing pulmonary disease. The ATS diagnostic criteria were designed for infections with M. avium, M. kansasii and M. abscessus; they may be less applicable to M. malmoense. Because of the high degree of true pulmonary M. malmoense infections observed, judgment on the clinical relevance of pulmonary $M$. malmoense isolates could probably be based on less strict criteria, as is advocated for M. kansasii [3], to prevent a prolonged period of inadequate treatment. The high degree of clinical relevance is in accordance with previous observations from northern Europe, varying from 70 to $84 \%$ using either ATS criteria or a modification of these criteria [11-14]. 


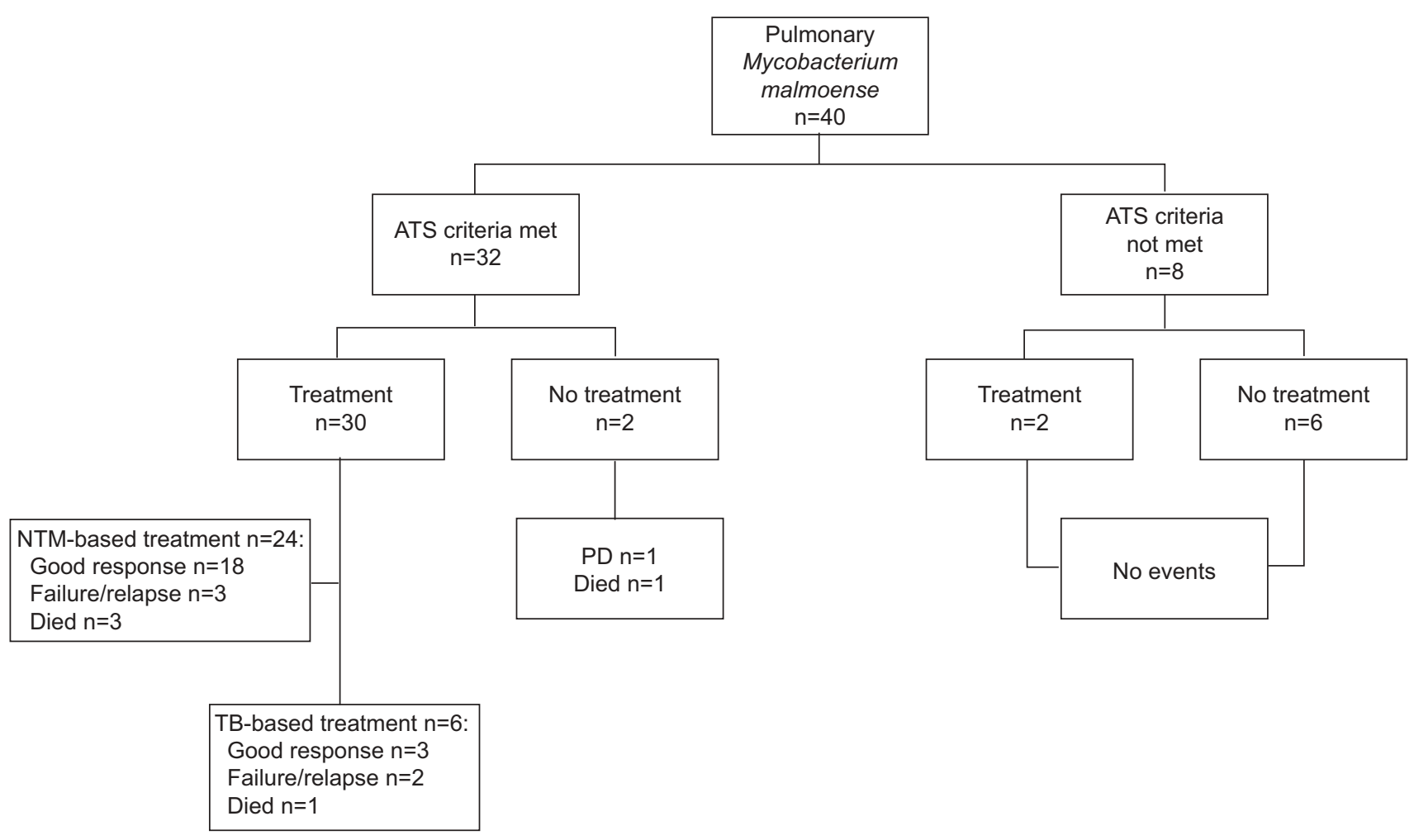

FIGURE 1. Frequency, treatment and outcome of pulmonary Mycobacterium malmoense disease in the Netherlands between 2002 and 2006 . ATS: American Thoracic Society; NTM: nontuberculosis mycobacteria; PD: progressive disease; TB: tuberculosis.

Interestingly, a dramatically lower clinical relevance of $10 \%$ was found in a retrospective case study of 73 patients in the USA [15]. There is no explanation for this difference; however, it suggests less pathogenic strains of M. malmoense in northern America compared with northern Europe. There are no known bacterial virulence factors for $M$. malmoense. Phylogenetically related $M$. szulgai and $M$. kansasii, both of which are suggested to be among the most pathogenic NTM [3,9], are known to harbour a region of difference-1-like genetic element (including esat- 6 and $c f p-10$ genes), which is a well known virulence factor for M. tuberculosis [16]. M. malmoense, however, lacks this element [17]. To date, immunological studies have focused on M. avium and $M$. abscessus $[18,19]$, rather than $M$. malmoense. Studies of $M$. malmoense pathogenesis and virulence in murine models are warranted, as are studies on the role of host genetic factors in M. malmoense disease.

In a recently published retrospective study of the prevalence of all NTM in Ontario, Canada between 1997 and 2003, M. malmoense was not isolated [20]. This observation is in contrast with the increase in $M$. malmoense notification in Europe since 1980, including increasing numbers of countries reporting isolation of $M$. malmoense [21]. This contrast suggests environmental niches favouring transmission to humans in Europe. Whether this observation can be linked with the lower clinical relevance observed in the USA needs to be studied.

Human transmission has never been proven, even in a setting of geographic clustering of cases. In a study published by DoIG et al. [22], small differences observed using pulsed-field electrophoresis were sufficient to show a lack of correlation between strain type and epidemiological or patient characteristics, making person-to-person spread unlikely.

The BTS treatment guidelines for pulmonary $M$. malmoense disease were not well adhered to, including choice of antimycobacterial therapy and duration of therapy. Treatment of M. malmoense disease was often preceded by or consisted only of tuberculosis treatment. This observation reflects the similar clinical presentation of pulmonary $M$. malmoense and M. tuberculosis complex infection and is a cause of concern. Increasing the use of PCR to rule out $M$. tuberculosis, providing a quick and definite NTM diagnosis, will probably decrease morbidity and mortality and prevent initiation of unnecessary contact tracing studies. Although hampered by our limited study group size and duration of follow-up, successful clinical response $(83 \%)$ in the current study in the optimally treated group is comparable with a $75 \%$ successful outcome found by HENRY et al. [23]. The failure and relapse rates found in the recently published BTS trial $12 \%$ in the group treated with rifampicin and ethambutol, $5 \%$ in the group treated with rifampicin and ethambutol combined with clarithromycine or ciprofloxacin) are lower compared with the rate found in our study (17\%) [24]. The shorter mean duration of antimycobacterial therapy probably negatively influenced treatment outcome in our population.

The mortality rate $(13 \%)$ found among adequately treated patients in the present study is comparable to that found by BANKS et al. [11] (15\%), and HENRY et al. [23] (11\%). The NTM disease-related mortality after 5 yrs of follow-up was found to be low (3.6\%) [24]. Mortality has been related to the length of 
delay between the diagnosis and start of treatment, while the occurrence of relapse has previously been associated with the total time-span of treatment [11]. Other factors suggested to independently affect mortality are in vitro resistance to ethambutol and the involvement of more than one lung zone [15]. In our study population, there was no significant association between ethambutol resistance and treatment failure.

The recently published BTS trial showed no additional benefit of adjunctive clarithromycin or ciprofloxacin over the 24month regimen of rifampicin and ethambutol for pulmonary M. malmoense disease. The addition of clarithromycin led to more side-effects [24]. These data are clinically important considering the extent of adjunctive macrolide and/or fluoroquinolone use in our study group.

The frequency and types of extrapulmonary disease in our study are similar to those found in a survey in Sweden, in which $21 \%$ out of 221 patients had extrapulmonary isolates, mainly lymphadenitis [14]. Paediatric cases of cervicofacial lymphadenitis are most frequent and tend to affect children in a limited age range, which may be related to environmental exposures specific to this age category [25], or to the state of development of the immune system in children. Contrary to pulmonary disease, $M$. malmoense lymphadenitis is a relatively benign condition. Surgery is considered to be the optimal treatment and yields good results $[3,26]$.

Tenosynovitis due to $M$. malmoense is rare, although case reports are available in international literature [27]. Extrapulmonary infection with $M$. malmoense is rare and dissemination is only observed in patients with severely impaired immunity, although rarely in HIV / AIDS.

In conclusion, pulmonary M. malmoense isolation is clinically relevant in $80 \%$ of all patients in the Netherlands, reflecting a level of virulence unmatched by other NTM species. Pulmonary disease resembling tuberculosis and paediatric lymphadenitis are the most common types of $M$. malmoense disease. Some patients are incorrectly treated for tuberculosis for a lengthy period. We recommend the use of molecular diagnostic tools for every sample positive for mycobacteria, to enable quick initiation of adequate therapy. Treatment outcome is relatively favourable when compared with other NTM infections. Future studies are necessary to optimise treatment regimens and to discern host and pathogen factors determining virulence and transmission to humans.

\section{STATEMENT OF INTEREST}

None declared.

\section{REFERENCES}

1 Schröder KH, Juhlin I. Mycobacterium malmoense sp. nov. Int J Syst Bacteriol 1977; 27: 241-246.

2 Martín-Casabona N, Bahrmand AR, Bennedsen J, et al. Spanish Group for Non-Tuberculosis Mycobacteria. Non-tuberculous mycobacteria: patterns of isolation. A multi-country retrospective survey. Int J Tuberc Lung Dis 2004; 8: 1186-1193.

3 Griffith DE, Aksamit T, Brown-Elliot BA, et al. An official ATS/ IDSA statement: diagnosis, treatment, and prevention of nontuberculous mycobacterial diseases. Am J Respir Crit Care Med 2007; 175: 367-416.
4 Subcommittee of the Joint Tuberculosis Committee of the British Thoracic Society. Management of opportunistic mycobacterial infections: Joint Tuberculosis Committee guidelines 1999. Thorax 2000; 55: 210-218.

5 Portaels F. Epidemiology of mycobacterial diseases. Clin Dermatol 1995; 13: 207-222.

6 Primm TP, Lucero CA, Falkinham III JO. Health impacts of environmental mycobacteria. Clin Microbiol Rev 2004; 17: 98-106.

7 The Research Committee of the British Thoracic Society. Pulmonary disease caused by M. malmoense in HIV negative patients: 5-yr follow up of patients receiving standardised treatment. Eur Respir J 2003; 21: 478-482.

8 van Klingeren B, Dessens-Kroon M, van der Laan T, et al. Drug susceptibility testing of Mycobacterium tuberculosis complex by use of a high-throughput, reproducible, absolute concentration method. J Clin Microbiol 2007; 45: 2662-2668.

9 van Ingen J, Boeree MJ, de Lange WCM, et al. Clinical relevance of Mycobacterium szulgai in the Netherlands. Clin Infect Dis 2008; 46: 1200-1205.

10 van Ingen J, Boeree MJ, de Lange WCM, et al. Mycobacterium xenopi clinical relevance and determinants, the Netherlands. Emerg Infect Dis 2008; 14: 385-389.

11 Banks J, Jenkins PA, Smith AP. Pulmonary infection with Mycobacterium malmoense, a review of treatment and response. Tubercle 1985; 66: 197-203.

12 Bollert FGE, Watt B, Greening AP, et al. Non-tuberculous pulmonary infections in Scotland: a cluster in Lothian? Thorax 1995; 50: 188-190.

13 Thomson VØ, Andersen $\AA$ B , Miörner H. Incidence and clinical significance of non-tuberculous mycobacteria isolated from clinical specimens during a 2-y nationwide survey. Scand J Infect Dis 2002; 34: 648-653.

14 Henriques B, Hoffner SE, Petrini B, et al. Infection with Mycobacterium malmoense in Sweden: report of 221 cases. Clin Infect Dis 1994; 18: 596-600.

15 Buchholz UT, McNeil MM, Keyes LE, et al. Mycobacterium malmoense infections in the United States, January 1993 through June 1995. Clin Infect Dis 1998; 27: 551-558.

16 Lewis KN, Liao R, Guinn KM, et al. Deletion of RD1 from Mycobacterium tuberculosis mimics Bacille Calmette-Guerin attenuation. J Infect Dis 2003; 187: 117-123.

17 Sorensen AL, Nagai S, Houen G, et al. Purification and characterization of a low-molecular-mass T-cell antigen secreted by Mycobacterium tuberculosis. Infect Immun 1995; 63: 1710-1717.

18 Rottman M, Catherinot E, Hochedez P, et al. Importance of T cells, gamma interferon, and tumor necrosis factor in immune control of the rapid grower Mycobacterium abscessus in C57BL/6 mice. Infect Immun 2007; 75: 5898-5907.

19 Doherty TM, Sher A. Defects in cell-mediated immunity affect chronic, but not innate, resistance of mice to Mycobacterium avium infection. J Immunol 1997; 158: 4822-4831.

20 Marras TK, Chedore P, Ying AM, et al. Isolation prevalence of pulmonary non-tuberculous mycobacteria in Ontario, 1997-2003. Thorax 2007; 62: 661-666.

21 Hoefsloot W, Boeree MJ, van Ingen J, et al. The rising incidence and clinical relevance of Mycobacterium malmoense. Int J Tuberc Lung Dis 2008; 12: 987-993.

22 Doig C, Muckersie L, Watt B, et al. Molecular epidemiology of Mycobacterium malmoense infections in Scotland. J Clin Microbiol 2002; 40: 1103-1105.

23 Henry MT, Inamdar L, O'Riordain D, et al. Nontuberculous mycobacteria in non-HIV patients: epidemiology, treatment and response. Eur Respir J 2004; 23: 741-746.

24 Jenkins PA, Campbell IA, Banks J, et al. Clarithromycin vs ciprofloxacin as adjuncts to rifampicin and ethambutol in the treatment of opportunist mycobacterial pulmonary diseases and an assessment of the value of immunotherapy with $M$. vaccae: 
a pragmatic, randomised trial by The British Thoracic Society. Thorax 2008; 63: 627-634.

25 Lindeboom JA, Prins JM, Bruijnesteijn van Coppenraet ES, et al. Cervicofacial lymphadenitis in children caused by Mycobacterium haemophilum. Clin Infect Dis 2005; 41: 1569-1575.

26 Lindeboom JA, Kuijper EJ, Bruijnesteijn van Coppenraet ES, et al. Surgical excision versus antibiotic treatment for nontuberculosis mycobacterial cervicofacial lymphadenitis in children: a multicenter, randomized, controlled trial. Clin Infect Dis 2007; 44: 10571064.

27 Olsen RJ, Cernoch PL, Land GA. Mycobacterial synovitis caused by slow-growing nonchromogenic species: eighteen cases and a review of the literature. Arch Pathol Lab Med 2006; 130: 783-791. 